\title{
Clinical Application and Feasibility of MRI-Guided Breast Biopsy of Breast Minimal Lesions in Chinese Population
}

\begin{abstract}
Jie Wang ${ }^{1 \dagger}$, Ying Song ${ }^{2 \dagger}$, Jiaqi Liu ${ }^{3 \dagger}$, Xiangzhi Meng ${ }^{3}$, Zeyu Xing ${ }^{3}$, Menglu Zhang ${ }^{3}$, Feng $\mathrm{Ye}^{2}$, Xin Wang ${ }^{3 *}$ and Xiang Wang ${ }^{3 *}$

${ }^{1}$ Department of Ultrasound, National Cancer Center/National Clinical Research Center for Cancer/Cancer Hospital, Chinese Academy of Medical Sciences and Peking Union Medical College, Beijing, China, ${ }^{2}$ Department of Diagnostic Radiology, National Cancer Center/National Clinical Research Center for Cancer/Cancer Hospital, Chinese Academy of Medical Sciences and Peking Union Medical College, Beijing, China, ${ }^{3}$ Department of Breast Surgical Oncology, National Cancer Center/National Clinical Research Center for Cancer/Cancer Hospital, Chinese Academy of Medical Sciences and Peking Union Medical College, Beijing, China
\end{abstract}

OPEN ACCESS

Edited by:

Roger M. Bourne

University of Sydney, Australia

Reviewed by:

Rossella Canese,

Istituto Superiore di Sanità (ISS), Italy

Wei Fu,

Johns Hopkins University,

United States

${ }^{*}$ Correspondence:

Xin Wang

xinwang@vip.126.com

Xiang Wang

xiangw@vip.sina.com

†These authors have contributed equally to this work

Specialty section

This article was submitted to Cancer Imaging and Image-directed Interventions, a section of the journa Frontiers in Oncology

Received: 11 July 2019 Accepted: 14 February 2020 Published: 06 March 2020

Citation:

Wang J, Song Y, Liu J, Meng X, Xing Z, Zhang $M$, Ye F, Wang $X$ and Wang X (2020) Clinical Application and Feasibility of MRI-Guided Breast Biopsy of Breast Minimal Lesions in

Chinese Population

Front. Oncol. 10:257.

doi: 10.3389/fonc.2020.00257
Objectives: Some breast lesions are not visible on mammography or ultrasonography, and magnetic resonance imaging (MRI) become the only way to monitor these lesions. The purpose of this study was to evaluate the clinical application of MRI-guided biopsy and MRI-guided wire localization of breast minimal lesions in Chinese population.

Methods: We evaluated 95 patients (the most patients of known in China) from August 2013 to December 2017. All the patients were scanned with a 1.5-Tesla MRI system (GE Medical Systems, America) in the prone position using a bilateral 8-channel phased-array breast coil and underwent MRI-guided wire localization or MRI-guided biopsy.

Results: MRI-guided wire localization and MRI-guided biopsy were successfully performed in 87 patients with 88 lesions (100\%, 88/88). After biopsy or surgery, 36 of 88 lesions (40.91\%) were malignant, and 52 of 88 lesions (59.09\%) were benign. Thirty-nine of 88 lesions (44.32\%) were masses, and 49 of 88 (55.68\%) showed non-mass enhancement. Statistical analysis showed there was no significant correlation between the malignancy rate and the type of lesion on MRI $(P=0.27)$. In this study, the rate of malignancy for Breast Imaging-Reporting and Data System (BI-RADS) 5 lesions was $100 \%$ (2 of 2) compared with $44.44 \%$ for BI-RADS 4C lesions (4 of 9), $42.42 \%$ for BI-RADS 4B lesions (14 of 33), and $36.36 \%$ for BI-RADS 4A lesions (16 of 44).

Conclusions: MRl-guided wire localization with subsequent surgical biopsy and MRI-guided biopsy are safe and effective tools for breast minimal lesions.

Keywords: breast cancer, minimal lesions, MRI-guided, biopsy, localization

\section{INTRODUCTION}

Breast cancer is one of the most common cancer diagnosed among women worldwide, accounting for the second leading cause of cancer-related death among women in US and the sixth in Chinese women (1-3). Magnetic resonance imaging (MRI) is widely used as a diagnostic tool for breast imaging in daily practice due to its high sensitivity for detecting primary, recurrent, and residual breast cancer. Some breast lesions are not visible on mammography 
or ultrasonography, and MRI can be used to regularly monitor these lesions. For suspicious breast lesions that are visible only on MRI, MRI-guided breast biopsy and MRI-guided wire localization with subsequent surgical biopsy are necessary for diagnosis and excluding multi-foci lesions in breast conserving surgery. Although MRI has a high reported sensitivity for detecting breast cancer of $94-100 \%$, its specificity is relatively low, ranging from 37 to $72 \%(4,5)$; thus, biopsy is often required to establish a diagnosis (6). These techniques have been performed successfully and evolved into routine methods in
Caucasian populations after $\sim 20$ years of clinical use $(7-10)$. However, there are inadequate studies in Asian countries about MRI-guided biopsy (1, 11, 12), though MRI-guided biopsy is more valuable in China compared with ultrasound-guided biopsy or mammogram-guided biopsy because there are more dense areas in glandular tissue of Asian women. Therefore, we report our initial clinical experience with MRI-guided wire localization with subsequent surgical biopsy and MRI-guided biopsy of breast minimal lesions only visible on MRI in Chinese women.

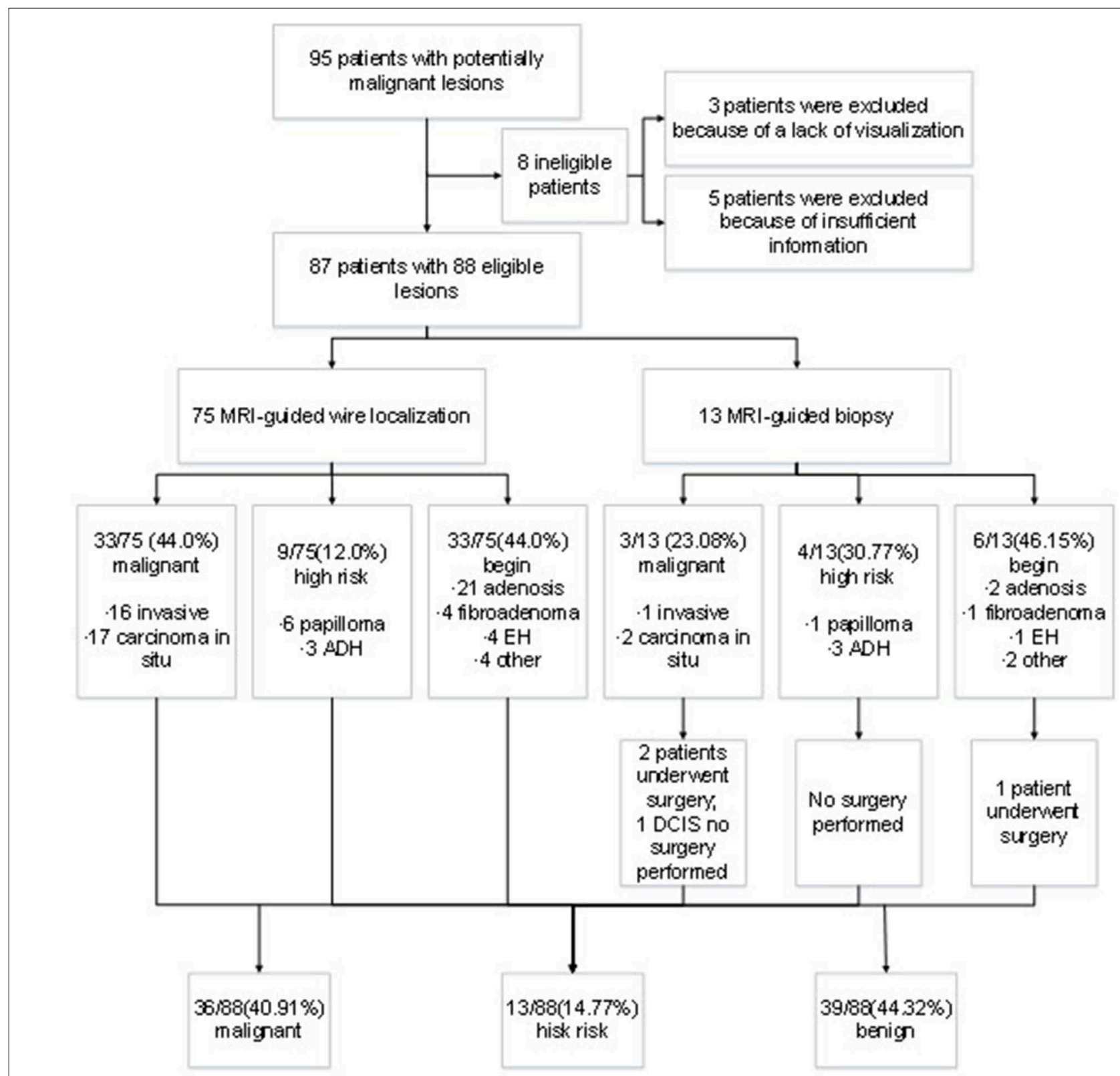

FIGURE 1 | Flowchart of lesion outcomes. 


\section{METHODS}

\section{Patient Population}

This retrospective study was approved by the institutional review board of National Cancer Center/National Clinical Research Center for Cancer/Cancer Hospital, Chinese Academy of Medical Sciences and Peking Union Medical College, Beijing, China. We evaluated all the patients who underwent MRI-guided wire localization with subsequent surgical biopsy or MRI-guided breast biopsy from August 2013 to December 2017. Following the explanation of risks, benefits and proposed procedure, informed consent was obtained. All the examinations were repeated every 3-6 months within 1 year, every 6-12 months thereafter.

\section{MRI Acquisition Parameters}

All the patients were scanned with a GE 1.5-Tesla MRI system in the prone position using a bilateral 8-channel phasedarray breast coil. The target lesion site was disinfected before scanning, and the breast was fixed with a special puncture frame. MRI scans were acquired using the following sequences with an Achieve scanner: vibrant sequence; axial view; field of view, $36-38 \mathrm{~cm}$; matrix size, $320 \times 288$; flip angle, 15; repetition time/echo time, $3.7 / 1.8 \mathrm{~ms}$; slice thickness, $2.6 \mathrm{~mm}$; and bandwidth $83.3 \mathrm{kHz}$. Unenhanced scans were performed before injection of a contrast agent. Continuous scans were performed without interruption $15 \mathrm{~s}$ after injection of the contrast agent. Using a high-pressure syringe, gadoliniumdiethylenetriamine pentaacetic acid (Magnevist; Schering, Berlin, Germany) at a dose of $0.2 \mathrm{mmol} / \mathrm{kg}$ of body weight was injected at a velocity of $2.0 \mathrm{ml} / \mathrm{s}$ through the cubital vein. Approximately

TABLE 1 | Pathology of lesions evaluated with MRI-guided biopsy or MRI-guided wire localization with subsequent surgical biopsy.

\begin{tabular}{|c|c|c|c|c|c|c|}
\hline \multirow[t]{2}{*}{ Pathology } & \multicolumn{2}{|c|}{$\begin{array}{c}\text { MRI-guided } \\
\text { biopsy } \\
(n=13)^{\star}\end{array}$} & \multicolumn{2}{|c|}{$\begin{array}{l}\text { MRI-guided wire } \\
\text { localization with } \\
\text { subsequent } \\
\text { surgical biopsy } \\
\quad(n=75)^{\star}\end{array}$} & \multicolumn{2}{|c|}{ Total $(n=88)$} \\
\hline & $n$ & $\%$ & $n$ & $\%$ & $n$ & $\%$ \\
\hline Malignant & 3 & 23.08 & 33 & 44.00 & 36 & 40.91 \\
\hline Invasive carcinoma & 1 & 7.69 & 16 & 21.33 & 17 & 19.32 \\
\hline Carcinoma in situ & 2 & 15.38 & 17 & 22.67 & 19 & 21.59 \\
\hline High risk & 4 & 30.77 & 9 & 12.00 & 13 & 14.77 \\
\hline Papilloma & 1 & 7.69 & 6 & 8.00 & 7 & 7.95 \\
\hline $\mathrm{ADH}$ & 3 & 23.08 & 3 & 4.00 & 6 & 6.82 \\
\hline Benign & 6 & 46.15 & 33 & 44.00 & 39 & 44.32 \\
\hline Adenosis & 2 & 15.38 & 21 & 28.00 & 23 & 26.14 \\
\hline Fibroadenoma & 1 & 7.69 & 4 & 5.33 & 5 & 5.68 \\
\hline $\mathrm{EH}$ & 1 & 7.69 & 4 & 5.33 & 5 & 5.68 \\
\hline Other & 2 & 15.38 & 4 & 5.33 & 6 & 6.82 \\
\hline Total & 13 & 100 & 75 & 100 & 88 & 100 \\
\hline
\end{tabular}

${ }^{*}$ Number of tumors.

$A D H$, atypical ductal hyperplasia; EH, epithelial hyperplasia.
$20 \mathrm{ml}$ of $0.9 \%$ normal saline was then injected with the same velocity to flush the vein.

\section{MRI-Guided Wire Localization and Biopsy}

All interventions were performed by the same staff radiologist with more than 10 years of experience with breast imaging. All procedures were conducted with the same MRI equipment.

After the skin overlying the area of interest was prepared and draped in the usual sterile fashion, the patient was placed in a prone position in the 1.5-T MRI scanner supported with a dedicated biopsy compression device. Dynamic contrastenhanced MRI (DCE-MRI) of the breast was obtained after contrast administration. The lesion depth was calculated based on the coordinates of the fiducial marker and the lesion. The patient was withdrawn from the magnet with the breast remaining in compression. After local anesthesia with $1 \%$ lidocaine, a scalpel incision was made in the skin. After insertion of the stylet introducer assembly to the appropriate depth, the stylet was removed from the breast, leaving the introducer within the breast and was replaced with an obturator to assist in MRI confirmation of the lesion location. After confirmation of the needle position and removal of the obturator, a 10-G vacuumassisted biopsy (VAB) device was inserted into the introducer. Biopsy specimens were obtained, and the biopsy device was removed, followed by reinsertion of the obturator. A post-biopsy MRI was performed to determine if the lesion had been sampled. A compression bandage was wrapped around the patient's chest

TABLE 2 | Clinical characteristics of patients who underwent MRI-guided biopsy or MRI-guided wire localization with subsequent surgical biopsy.

\begin{tabular}{|c|c|c|c|c|c|}
\hline Clinical characteristic & $n$ & Benign (\%) & Malignant (\%) & $x^{2}$ & $p$ \\
\hline \multicolumn{6}{|l|}{ Age (years) } \\
\hline$\leq 40$ & 15 & $12 / 15(80.00)$ & 3/15 (20.00) & 5.00 & 0.08 \\
\hline 40-60 & 55 & 33/55 (60.00) & 22/55 (40.00) & & \\
\hline$\geq 60$ & 17 & $7 / 17$ (41.18) & 10/17 (58.82) & & \\
\hline \multicolumn{6}{|l|}{ Menstrual status } \\
\hline Premenopausal & 49 & $32 / 49$ (65.31) & 17/49 (34.69) & 1.43 & 0.23 \\
\hline Postmenopausal & 38 & 20/38 (52.63) & $18 / 38(47.37)$ & & \\
\hline \multicolumn{6}{|l|}{ Imaging characteristic } \\
\hline Mass & 39 & 26/39 (66.67) & 13/39 (33.33) & 1.21 & 0.27 \\
\hline $\begin{array}{l}\text { Nonmass-like } \\
\text { enhancement }\end{array}$ & 49 & $27 / 49(55.10)$ & $22 / 49(44.90)$ & & \\
\hline \multicolumn{6}{|l|}{ Tumor size (cm) } \\
\hline$\leq 1$ & 29 & $21 / 29(72.41)$ & 8/29 (27.59) & 7.60 & 0.55 \\
\hline $1-2$ & 29 & 17/29 (58.62) & $12 / 29(41.38)$ & & \\
\hline$\geq 2$ & 16 & 10/16 (62.50) & 6/16 (37.50) & & \\
\hline NA & 14 & 4/14 (28.57) & $10 / 14(71.43)$ & & \\
\hline \multicolumn{6}{|l|}{ Bi-RADS } \\
\hline $4 a$ & 44 & 28/44 (63.64) & $16 / 44(36.36)$ & 4.03 & 0.26 \\
\hline $4 b$ & 33 & 19/33 (57.58) & 14/33 (42.42) & & \\
\hline $4 c$ & 9 & $5 / 9(55.56)$ & $4 / 9(44.44)$ & & \\
\hline 5 & 2 & 0 & $2 / 2(100)$ & & \\
\hline
\end{tabular}

NA, not available. 
after the biopsy. To stop bleeding and to minimize the risk of hematoma formation, firm manual compression of the breast was performed for $10 \mathrm{~min}$. The same procedure was used for MRI-guided wire localization.

The pathological diagnosis of 87 patients was confirmed by three or four pathologists with rich experience, including the pathological types, immunohistochemical and lymph node status of all suspicious lesions.

\section{Data Collection and Statistical Analysis}

Eighty-eight suspicious lesions from 87 patients were retrospectively analyzed. This study included Breast ImagingReporting and Data System (BI-RADS) 4 or 5 lesions that were visible only on MRI. Clinical information including age, menstrual status, lesion location, histopathological results, and MRI characteristics of the targeted lesion (BI-RADS category, lesion size, and mass or non-mass enhancement) was recorded.

The chi-square test was used to compare lesion characteristics and histopathological results. SPSS version 15.0 (IBM, Chicago, USA) was used. The two-tailed $p<0.05$ was set as the limit of statistical significance.

\section{RESULTS}

A total of 95 patients underwent MRI-guided wire localization with subsequent surgical biopsy or MRI-guided breast biopsy from August 2013 to December 2017 at our institute. However, three cases had unsuccessful localization or biopsy attempts because of a lack of visualization of the initial biopsy target on the scheduled day of biopsy, and five cases were excluded from analysis for the lack of insufficient postoperative information. Ultimately, the remaining 87 patients were included into analysis. The outcomes of lesion was presented in Figure 1. The median age of patients was 50 years old ranging from 29 to 77 years old. There was no bleeding, hematoma, infection, or other adverse reactions after the MRI-guided biopsy. Meanwhile, no recurrent malignant breast tumor was found in all the patients during follow-up.

After biopsy or surgery, 36 of 88 lesions (40.91\%) were found to be malignant, and 52 of 88 (59.09\%) were benign. Seventeen of 88 lesions (19.32\%) were invasive mammary carcinoma, and $19(21.59 \%)$ were carcinoma in situ (Table 1). Of the 52 benign lesions, 23 of $88(26.14 \%)$ were breast adenosis, five (5.68\%) were epithelial hyperplasia (EH), five (5.68\%) were breast fibroadenomas, seven $(7.95 \%)$ were papillomas, six $(6.82 \%)$ were atypical ductal hyperplasia $(\mathrm{ADH})$, and six (6.82\%) were other benign lesions (Table 1). All the lesions had morphologic features suspicious for or highly suggestive of malignancy according to the BI-RADS category of MRI $(\mathrm{C} 4 \mathrm{a}=44, \mathrm{C} 4 \mathrm{~b}=33, \mathrm{C} 4 \mathrm{c}=9$, and $\mathrm{C} 5=2$ ). Clinical characteristics of breast cancer patients with the MRI-guided wire localization and MRI-guided biopsy are summarized in Table 2.

According the type of imaging, of the 88 lesions that were evaluated with MRI-guided biopsy (Figure 2) or localization
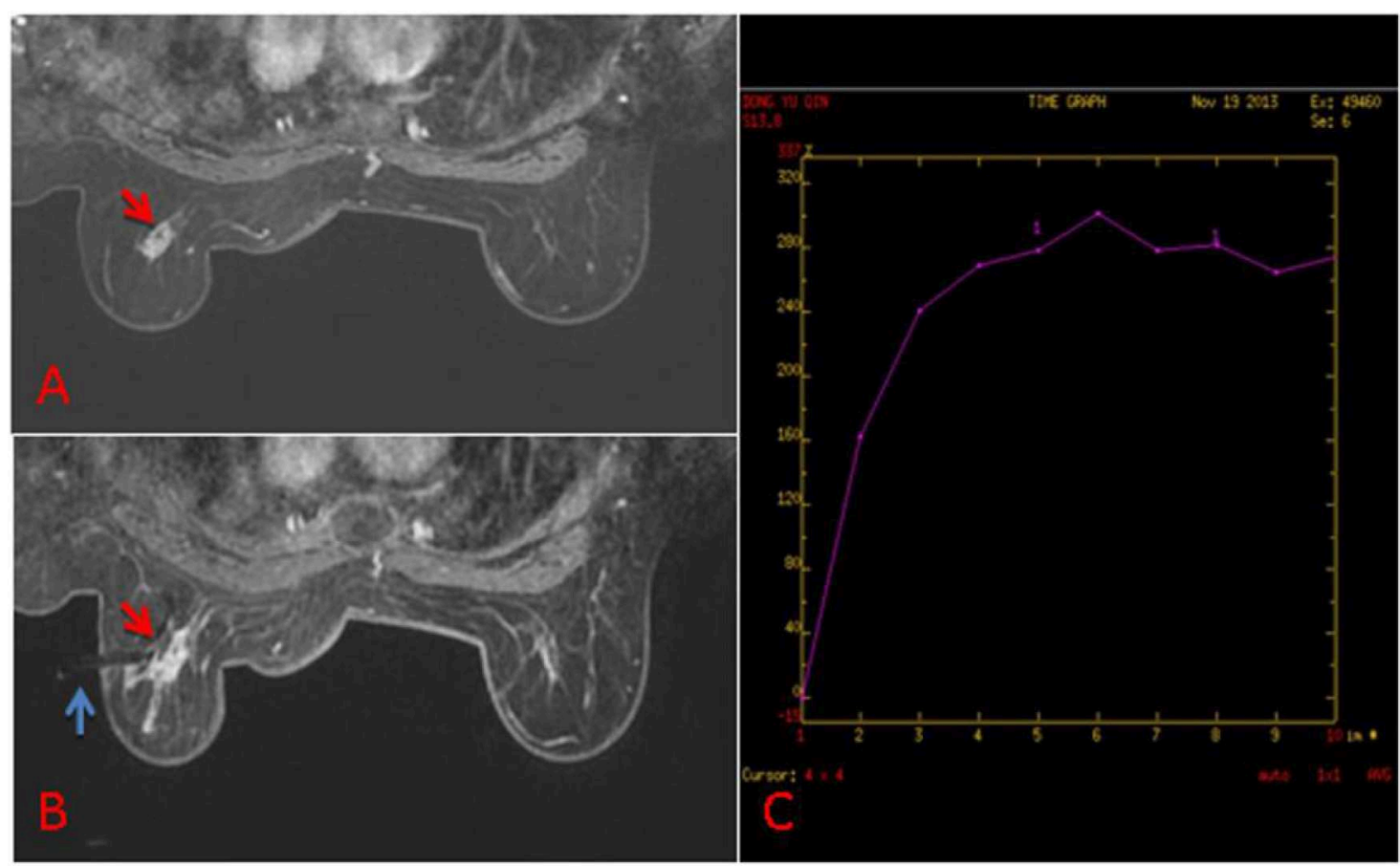

FIGURE 2 | (A) 63-year old woman, T1-weighted fat suppressed contrast enhanced MRI showed a irregular mass at the left breast, which was suspicion for malignancy, and the BI-RADS category was considered as level 5. It was not found by ultrasound or mammography. (B) The lesion (red arrow) was performed by MR-guided biopsy (blue arrow), and it was confirmed to be invasive ductal carcinoma. (C) The dynamic contrast-enhanced time-signal intensity curve (TIC) was appeared as "fast-plateau type". 

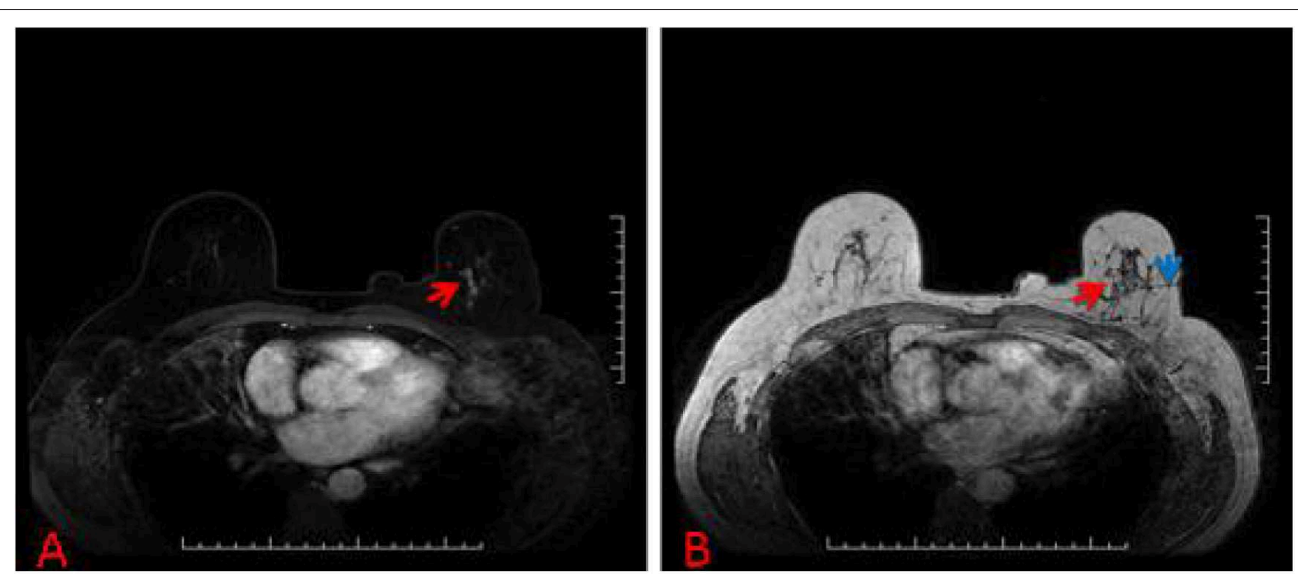

FIGURE 3 | (A) 57-year-old woman, T1-weighted fat suppressed contrast enhanced MRI showed a suspicious lesion at the left breast, and the BI-RADS category was considered as level 4C. (B) The lesion (red arrow) that was revealed to be invasive ductal carcinoma after MRI-guided wire localization (blue arrow) with subsequent surgical biopsy (we chose T1-weighted MRI in order to show the lesion and wire location more clearly).
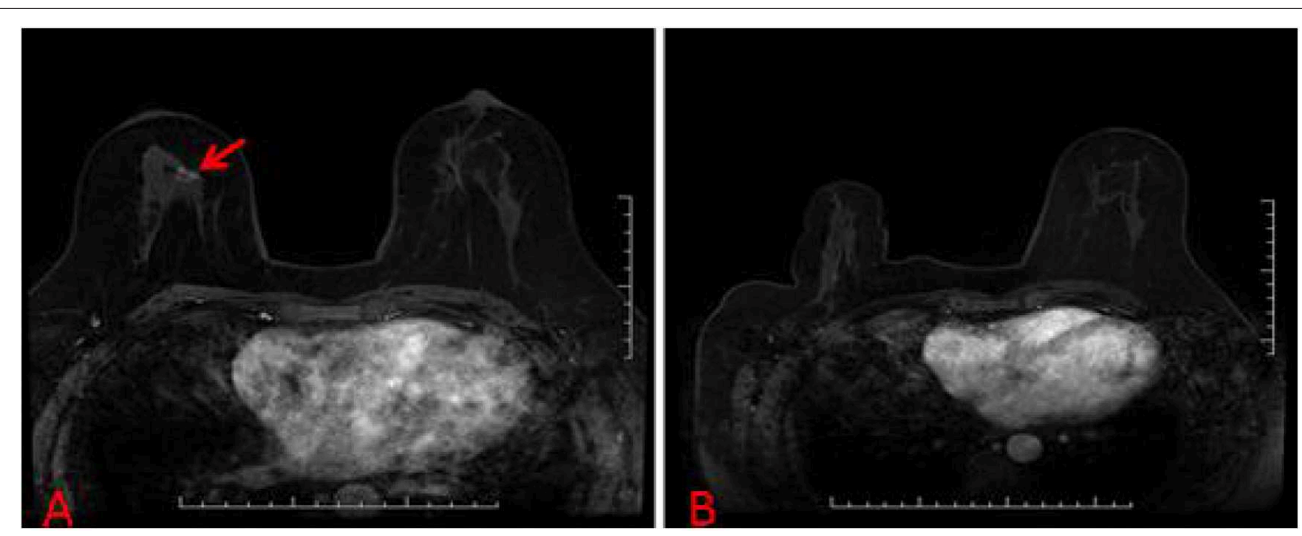

FIGURE 4 | (A) A suspicious breast lesions (red arrow) that are visible only on MRI. (B) The patient with unsuccessful localization attempts because of a lack of visualization of the initial biopsy target on the scheduled day of biopsy.

(Figure 3), 39 of $88(44.32 \%)$ were masses, including 13 malignant lesions and 26 benign lesions, and 49 of 88 (55.68\%) had non-mass enhancement, including 22 malignant lesions and 27 benign lesions. However, there was no significant association between the malignancy rate and the type of lesion on MRI ( $p$ $=0.27$ ). The mean size of all the lesions was $1.49 \pm 0.91 \mathrm{~cm}$; the mean lesion size was $1.57 \pm 0.89 \mathrm{~cm}$ in malignant lesions vs. $1.46 \pm 0.91 \mathrm{~cm}$ in benign lesions $(p=0.55)$. The probability of malignancy on MRI-guided biopsy or MRI-guided wire localization also increased with the BI-RADS category of the lesion of interest. In this study, the rate of malignancy for BIRADS 5 lesions was $100 \%$ ( 2 of 2), compared with $44.44 \%$ for BI-RADS 4C lesions (4 of 9), 42.42\% for BI-RADS 4B lesions (14 of 33 ), and $36.36 \%$ for BI-RADS 4 A lesions (16 of 44 ).

\section{DISCUSSION}

The first MRI-guided clinical trials were reported in 1986 (13). Since then, MRI-guided clinical applications have been successfully applied to lesions in various anatomical regions, such as pancreatic, breast, prostate, lung tumor, and renal tumor lesions (14-18). After $\sim 20$ years of clinical use, MRIguided breast biopsy has evolved into a routine method in Western European countries $(19,20)$. A small number of breast lesions cannot be detected by breast ultrasonography and mammography and can be identified only by MRI. Thus, the annual MRI scan is important for the breast cancer screening, especially for the women at high risk. However, according to the costs of more time and economy, MRI-guided procedures are reserved for lesions that are visible only on MRI and are judged to be suspicious and potential influence therapeutic decisions (21).

The malignancy rate in this study of women with MRI-visible breast lesions who underwent MRI-guided wire localization with subsequent surgical biopsy and MRI-guided biopsy of breast minimal lesions was $40.91 \%$ (in 13 patients who underwent MRI-guided biopsy, the malignancy rate was $23.08 \%$, and in 74 patients with 75 eligible lesions who underwent MRI-guided wire localization with surgical biopsy, the malignancy rate was $44.0 \%$ ), which is higher than the reported range of $20-35 \%(22-25)$. The main difference in this study was that the majority of patients ( $n$ $=74$ ) underwent MRI-guided wire localization with subsequent 
surgical biopsy (the number of suspicious lesions was 75), and only 13 patients underwent MRI-guided biopsy. There may be several reasons for this phenomenon in this Chinese population. The most important reason is that there is currently no clinical experience MRI-guided breast biopsy in China, thus, Chinese patients may be more anxious compared with patients in Western countries, and when an occult lesion was found on MRI, most Chinese patients chose surgical treatment. Other reasons like the possible false negative rate of needle biopsy, the high selfpaying price of MRI-guided biopsy also push breast surgeons and patients to chose MRI-guided wire localization with subsequent surgical biopsy. Thus, the majority of patients underwent MRIguided wire localization with subsequent surgical biopsy in this study.

MRI-guided biopsies have some chance of being canceled, and the reported rates of biopsy cancellation range from 8 to $13 \%$; most often biopsies are canceled because of a lack of visualization of the initial biopsy target on the scheduled day of biopsy (26$28)$. In this study, three lesions $(3.33 \%, 3 / 90)$ had unsuccessful localization or biopsy attempts because of a lack of visualization on the day of examination (Figure 4), but this rate is lower than the previously reported rate. These canceled biopsies might be a source of confusion and frustration for both clinicians and patients because of the small possibility of a missed malignancy, so a follow-up MRI at 6 months is recommended. Some studies have reported specifically on subsequent malignancies in patients with canceled MRI-guided biopsies (27-29). In the three patients who had canceled MRI-guided biopsies, no malignant breast tumors were found during follow-up until now.

There is currently no unified conclusion about the association between lesion morphology and malignancy rate. In our study, $55.68 \%$ (49 of 88 ) of the lesions had non-mass enhancement, and the cancer detection rate was $44.90 \%$ (22 of 49 ). The other lesions were masses, and the malignancy rate was 33.33\% (13 of 39), which was lower than that for non-mass enhancing lesions. Rauch et al. (30) reported a greater malignancy rate for areas of non-mass enhancement (34\%, 22 of 76) compared with masses (22\%, 22 of 17). However, Han et al. (10) reported no significant difference in the malignancy rate of MRI-biopsied masses (34\%) compared with that in non-mass enhancing lesions (27\%).

Our research had some limitations. Firstly, though this is the largest sample study assessing the MRI-guided wire localization with subsequent surgical biopsy or MRI-guided breast biopsy in China, it is still limited by its relative small sample size and retrospective nature. Secondly, we did not correlate clinical stage and hormone receptors with imaging features. Finally, we did not consider the effect of breast size on the success rate of MRI-guided examination. Due to technical reasons, we rejected some patients with small breast volume in the early stage. With the accumulation of experience, fewer and fewer patients were excluded for this reason. In conclusion, large sample prospective studies which involve more information are warranted in the future.

Compared with ultrasound-guided biopsy or mammogramguided biopsy, MRI-guided biopsy is more valuable in China because there are more dense areas in glandular tissue of Asian women. In addition, the rate of breast-conserving therapy in china is lower than that in Europe and American, the use of MRI-guided biopsy of breast minimal lesions could increase the rate of breast-conserving therapy in china. However, there are only few medical centers could performed this procedure due to lack of Asian specific breast coil which ought be smaller than Western one as well as insurance does not cover such procedure. Therefore, this study has an initiating significance in China.

\section{CONCLUSION}

In conclusion, MRI-guided wire localization and MRI-guided biopsy can be safely, easily and effectively performed in occult breast lesions without major complications. After MRI-guided biopsy, follow-up within 6 months may be recommended because of the opportunity of underestimation of the disease. For occult lesions, MRI-guided biopsy is the only method that can confirm the diagnosis of suspicious enhancing breast lesions; however, this technique does pose some challenges.

\section{DATA AVAILABILITY STATEMENT}

The raw data supporting the conclusions of this article will be made available by the authors, without undue reservation, to any qualified researcher.

\section{ETHICS STATEMENT}

Ethical review and approval was not required for the retrospective study on human participants in accordance with the local legislation and institutional requirements. The patients/participants provided their written informed consent to participate in this study.

\section{AUTHOR CONTRIBUTIONS}

JW wrote the manuscript and performed the statistical analysis. YS and JL contributed conception and design of the study. XM, ZX, and MZ organized the database. FY, XinW, and XiaW wrote sections of the manuscript. All authors contributed to manuscript revision, read, and approved the submitted version.

\section{FUNDING}

This research was funded in part by the National Natural Science Foundation of China (81602343 to XinW), the CAMS Initiative Fund for Medical Sciences (2016-I2M-1-001 to XiaW, 2017-I2M-3-004 to XinW), the Non-profit Central Research Institute Fund of Chinese Academy of Medical Sciences (2017PT32001 and 2016ZX310178 to XinW), the Beijing Hope Run Special Fund (LC2017B15 to XinW, LC2017B16 to ZX), and the Capital's Funds for Health Improvement and Research (2016-4-4026 to XinW).

\section{ACKNOWLEDGMENTS}

We are very grateful to everyone involved in this research who generously helped us with our thesis. 


\section{REFERENCES}

1. DeSantis CE, Fedewa SA, Goding SA, Kramer JL, Smith RA, Jemal A. Breast cancer statistics, 2015: convergence of incidence rates between black and white women. CA Cancer J Clin. (2016) 66:31-42. doi: 10.3322/caac.21320

2. Fan L, Strasser-Weippl K, Li JJ, St Louis J, Finkelstein DM, Yu $\mathrm{KD}$, et al. Breast cancer in China. Lancet Oncol. (2014) 15:e279-89. doi: 10.1016/S1470-2045(13)70567-9

3. Chen W, Zheng R, Baade PD, Zhang S, Zeng H, Bray F, et al. Cancer statistics in China, 2015. CA Cancer J Clin. (2016) 66:115-32. doi: 10.3322/caac.21338

4. Wang HY, Zhao YN, Wu JZ, Wang Z, Tang JH. MRI-guided wire localization open biopsy is safe and effective for suspicious cancer on breast MRI. Asian Pac J Cancer Prev. (2015) 16:1715-8. doi: 10.7314/apjcp.2015.16.5.1715

5. Saslow D, Boetes C, Burke W, Harms S, Leach MO, Lehman CD, et al. American cancer society guidelines for breast screening with MRI as an adjunct to mammography. CA Cancer J Clin. (2007) 57:75-89. doi: $10.3322 /$ canjclin.57.2.75

6. Peters NH, Borel Rinkes IH, Zuithoff NP, Mali WP, Moons KG, Peeters PH. Meta-analysis of MR imaging in the diagnosis of breast lesions. Radiology. (2008) 246:116-24. doi: 10.1148/radiol.2461061298

7. Imschweiler T, Haueisen H, Kampmann G, Rageth L, Seifert B, Rageth C, et al. MRI-guided vacuum-assisted breast biopsy: comparison with stereotactically guided and ultrasound-guided techniques. Eur Radiol. (2014) 24:128-35. doi: 10.1007/s00330-013-2989-5

8. Brennan SB. Breast magnetic resonance imaging for the interventionalist: magnetic resonance imaging-guided vacuum assisted breast biopsy. Tech Vasc Interv Radiol. (2014) 17:40-8. doi: 10.1053/j.tvir.2013.12.007

9. Heywang-Köbrunner SH, Sinnatamby R, Lebeau A, Lebrecht A, Britton PD, Schreer I. Interdisciplinary consensus on the uses and technique of MRguided vacuum-assisted breast biopsy (VAB): results of a European consensus meeting. Eur J Radiol. (2009) 72:289-94. doi: 10.1016/j.ejrad.2008.07.010

10. Han BK, Schnall MD, Orel SG, Rosen M. Outcome of MRI-guided breast biopsy. AJR Am J Roentgenol. (2008) 191:1798-804. doi: 10.2214/AJR.07.2827

11. Tozaki M, Yamashiro N, Sakamoto $M$, Sakamoto N, Mizuuchi N, Fukuma E. Magnetic resonance-guided vacuum-assisted breast biopsy: results in 100 Japanese women. Jpn J Radiol. (2010) 28:527-33. doi: 10.1007/s11604-010-0464-7

12. Tozaki M, Yamashiro N, Suzuki T, Kawano N, Ozaki S, Sakamoto N, et al. MR-guided vacuum-assisted breast biopsy: is it an essential technique? Breast Cancer. (2009) 16:121-5. doi: 10.1007/s12282-008-0074-8

13. Mueller PR, Stark DD, Simeone JF, Saini S, Butch RJ, Edelman RR, et al. MR guided aspiration biopsy: needle design and clinical trials. Radiology. (1986) 161:605-9. doi: 10.1148/radiology.161.3.3786706

14. Liu C, Wang L, He X, Xu Y, Lu D, Li P, et al. 1.0T MR-guided percutaneous coaxial cutting needle biopsy in pancreatic lesion diagnosis. J Magn Reson Imaging. (2018) 48:382-8. doi: 10.1002/jmri.25952

15. Ahrar K, Ahrar JU, Javadi S, Pan L, Milton DR, Wood CG, et al. Real-time magnetic resonance imaging-guided cryoablation of small renal tumors at 1.5 T. Invest Radiol. (2013) 48:437-44. doi: 10.1097/RLI.0b013e31828027c2

16. Verheyden C, Pages-Bouic E, Balleyguier C, Cherel P, Lepori D, Laffargue $\mathrm{G}$, et al. Underestimation rate at MR imaging-guided vacuum-assisted breast biopsy: a multi-institutional retrospective study of 1509 breast biopsies. Radiology. (2016) 281:708-19. doi: 10.1148/radiol.2016151947

17. Liu M, Huang J, Xu Y, He X, Li L, Lü Y, et al. MR-guided percutaneous biopsy of solitary pulmonary lesions using a 1.0-T open high-field MRI scanner with respiratory gating. Eur Radiol. (2017) 27:1459-66. doi: 10.1007/s00330-016-4518-9
18. Gangi A, Tsoumakidou G, Abdelli O, Buy X, de Mathelin M, Jacqmin D, et al. Percutaneous MR-guided cryoablation of prostate cancer: initial experience. Eur Radiol. (2012) 22:1829-35. doi: 10.1007/s00330-012-2411-8

19. Krug B, Hellmich M, Ulhaas A, Krämer S, Rhiem K, Zarghooni V, et al. Vacuum-assisted breast Biopsies (VAB) carried out on an open 1.0T MR imager: influence of patient and target characteristics on the procedural and clinical results. Eur J Radiol. (2016) 85:1157-66. doi: 10.1016/j.ejrad.2016.02.030

20. Mann RM, Kuhl CK, Kinkel K, Boetes C. Breast MRI: guidelines from the European society of breast imaging. Eur Radiol. (2008) 18:1307-18. doi: 10.1007/s00330-008-0863-7

21. Sardanelli F, Boetes C, Borisch B, Decker T, Federico M, Gilbert FJ, et al. Magnetic resonance imaging of the breast: recommendations from the EUSOMA working group. Eur J Cancer. (2010) 46:1296-316. doi: 10.1016/j.ejca.2010.02.015

22. Myers KS, Kamel IR, Macura KJ. MRI-guided breast biopsy: outcomes and effect on patient management. Clin Breast Cancer. (2015) 15:143-52. doi: 10.1016/j.clbc.2014.11.003

23. Taşkin F, Soyder A, Tanyeri A, Öztürk VS, Ünsal A. Lesion characteristics, histopathologic results, and follow-up of breast lesions after MRI-guided biopsy. Diagn Interv Radiol. (2017) 23:333-8. doi: 10.5152/dir.2017. 17004

24. Spick C, Schernthaner M, Pinker K, Kapetas P, Bernathova M, Polanec $\mathrm{SH}$, et al. MR-guided vacuum-assisted breast biopsy of MRI-only lesions: a single center experience. Eur Radiol. (2016) 26:3908-16. doi: 10.1007/s00330-016-4267-9

25. Ferré R, Ianculescu V, Ciolovan L, Mathieu MC, Uzan C, Canale S, et al. Diagnostic performance of MR-guided vacuum-assisted breast biopsy: 8 years of experience. Breast J. (2016) 22:83-9. doi: 10.1111/tbj.12519

26. Pinnamaneni N, Moy L, Gao Y, Melsaether AN, Babb JS, Toth HK, et al. Canceled MRI-guided breast biopsies due to nonvisualization: follow-up and outcomes. Acad Radiol. (2018) 25:1101-10. doi: 10.1016/j.acra.2018.01.016

27. Niell BL, Lee JM, Johansen C, Halpern EF, Rafferty EA. Patient outcomes in canceled MRI-guided breast biopsies. AJR Am J Roentgenol. (2014) 202:223-8. doi: 10.2214/AJR.12.10228

28. Hefler L, Casselman J, Amaya B, Heinig A, Alberich T, Koelbl H, et al. Follow-up of breast lesions detected by MRI not biopsied due to absent enhancement of contrast medium. Eur Radiol. (2003) 13:344-6. doi: 10.1007/s00330-002-1713-7

29. Brennan SB, Sung JS, Dershaw DD, Liberman L, Morris EA. Cancellation of MR imaging-guided breast biopsy due to lesion nonvisualization: frequency and follow-up. Radiology. (2011) 261:92-9. doi: 10.1148/radiol.11100720

30. Rauch GM, Dogan BE, Smith TB, Liu P, Yang WT. Outcome analysis of 9gauge MRI-guided vacuum-assisted core needle breast biopsies. AJR Am J Roentgenol. (2012) 198:292-9. doi: 10.2214/AJR.11.7594

Conflict of Interest: The authors declare that the research was conducted in the absence of any commercial or financial relationships that could be construed as a potential conflict of interest.

Copyright (c) 2020 Wang, Song, Liu, Meng, Xing, Zhang, Ye, Wang and Wang. This is an open-access article distributed under the terms of the Creative Commons Attribution License (CC BY). The use, distribution or reproduction in other forums is permitted, provided the original author(s) and the copyright owner(s) are credited and that the original publication in this journal is cited, in accordance with accepted academic practice. No use, distribution or reproduction is permitted which does not comply with these terms. 